\section{Satellites to the fore}

\section{from W. H. McCrea}

EARTH's satellite the Moon is not only the astronomical body other than the Earth about which most is known; it is also the astronomical body about which knowledge has increased most rapidly in recent years--thanks to the spectacular successes of the Apollo and Luna missions. Even more recently, Pioneer 10 and 11 have provided invaluable new information about the Galilean satellites of Jupiter. At the present time, therefore, astronomers are more satellite-minded than ever before. Above all, they are interested in the origin of satellites because this may provide a check upon, or even a clue to, the origin of the planets. After all, astronomers have only the one planetary system to study, whereas they have six satellite systems; so it is reasonable to think that the satellite problem might be solved before the planet problem.

T. Gold has reviewed the situation and made a number of suggestions (Icarus, 25, 489-491: 1975). He begins by asserting that all planets for which satellite orbits would be stable do possess satellites, claiming that for Mercury the solar perturbation is too large. that the retrograde spin of Venus would cause satellites to spiral into the planet through tidal friction (but I think he means that the slowness of the spin of Venus would render it retrograde as seen by anv possible satellite), and not mentioning Pluto. Anvhow, Gold is obviously correct in remarking that satellites are a common feature of the Solar System. and this stimulates him to seek a process of formation of the widest possible applicability. In general terms, he appeals to the dvnamics of grains in the presence of massive gravitating bodies (Sun and planets) and of a resisting medium (neutral and charged particles and photons). If initially a lot of grains are moving at random in a gravitational field under the action of no other forces, the motion remains random, that is, it is conservative, and there is no tendency for the motion or space-distribution to become systematised in any way. But matters are different if dissipative forces play a part. If there are inelastic collisions between the grains themselves, then there may be focusing into the 'accretion streams' or 'jet streams' considered by some cosmogonists to be essential for the development of the Solar System. If there is a dissipative frictional drag exerted by a diffuse medium in the system, even at a stage when interaction between the grains is unimportant. there may be accumulation of grains in resonant bands in the gravitational field of the moving planets. Both types of behaviour may be included under the heading of gravitational focusing. The novelty of Gold's discussion is to suggest a crucial cosmogonic role for the second type.

In bald terms the notion is: In the 'early solar nebula' grains were plentiful from direct condensation and from collisions between larger bodies of material. Under the action of the general gravitational field and the 'drag' forces, a grain near the central plane would normally spiral inwards or outwards from the Sun. Such spiralling could, however, be halted or speeded up if the motion became resonant with that of an orbiting planet. Thus lanes of accumulation or avoidance would have been formed. Here Gold remarks in passing that a lane of accumulation could be favourable for 'snowballing'; he suggests this as an explanation of the asteroids in Trojan orbits. $\mathrm{He}$ remarks too that in regard to any particular lane at any particular epoch there could be selection with respect to grain size or grain composition.

The main suggestion is that, material having been concentrated into a welldefined lane by resonance effects, when the resonance was broken, such material continued in a well-defined stream though once more in spiral motion; further, when it encountered a planet, there was a significant probability of its being captured into a ring round the planet. There would have been numerous cases of this happening throughout the system. Gold sees no serious difficulty in the passage from grains in circumplanetary rings to larger bodies, and the subsequent coalescence of these into one or more satellites round a planet as we now know them.

The interest of Gold's essay. one ventures to think. is its demonstration that the prohlem of the orioin of satellites is still wide open. It was originally presented at a colloquium. It can scarcely have been intended to do more than throw out suggestions, for the treatment is non-quantitative throughout. This makes it difficult to comment further; however, when Gold writes, the step from circumplanetary rings . . . to the formation of satellite bodies is not a difficult one and has been discussed on many occasions' one is bound to remark that although this has indeed been discussed often enough, one is not aware that it has ever been established that satellite bodies must form in these circumstances. Also, although there is much attractiveness in Gold's implication that all the regular satellites might have been formed by one and the same general process, the very great differences between the properties of the main satellites and the minor (regular) satellites in the actual Solar System give strong indications of the operation of two distinct mechanisms of formation. Maybe we can look forward to a quantitative account of Gold's ideas in a form that could be more critically compared with observation.

\section{Nutrient balance during succession}

\section{from Peter D. Moore}

THE developmental growth of an ecosystem, commonly termed succession, involves a steadily increasing biomass, reaching a maximum at the climax, or steady state, which is ultimately attained. The increase in living material during succession places a demand upon the inorganic, abiotic component of the system for those elements necessary for the construction of living tissues. Ultimately the biotic component thus becomes an important reservoir of nutrients-a feature which may contribute very considerably to the overall nutrient capital of an ecosystem (see Nature, 254, 184; 1975). Actual data concerning the changing nutrient demand of the biotic component of an ecosystem during its development are, however, sadly lacking.

Experimental manipulation of watersheds in the Hubbard Brook Experimental Forest in New Hampshire, has provided a certain amount of information on which to speculate. Six small, undisturbed watersheds at this site have been studied intensively over a number of years and data concerning nutrient input from rainfall and output from stream drainage has been collected. In 1965 one of the forested watersheds ( $15.6 \mathrm{ha}$ in area) was clearfelled and the resulting changes in the chemistry of the runoff waters were subsequently reported by Bormann, Likens, Fisher and Pierce (Science, 159, 882; 1968). One of the most striking changes observed was the increased loss of nitrate nitrogen from the ecosystem following felling; a loss of $1.5 \mathrm{~kg} \mathrm{~N} \mathrm{ha}^{-1} \mathrm{yr}^{-1}$ occurred in a control forested watershed, whilst $58.1 \mathrm{~kg}$ $\mathrm{Nha}^{-1} \mathrm{yr}^{-1}$ were lost from the clearfelled watershed. Many cations also increased in concentration in the runoff water, thus permitting the conclusion that disturbance led to increased leaching and therefore a reduced capacity for nutrient conservation.

Marks and Bormann (Science, 176, 914; 1972) further demonstrated that revegetation of a disturbed area led to considerably diminished nutrient runoff and they concluded that the early stages of succession which were then 